\title{
Distal Doppler-guided transanal hemorrhoidal dearterialization with mucopexy versus conventional hemorrhoidectomy for grade III and IV hemorrhoids: postoperative morbidity and long-term outcomes
}

\author{
L. Trenti ${ }^{1,2} \cdot$ S. Biondo ${ }^{1,2} \cdot$ A. Galvez ${ }^{1,2} \cdot$ A. Bravo $^{1,2} \cdot$ J. Cabrera $^{3}$. \\ E. Kreisler ${ }^{1,2}$
}

Received: 2 February 2017 / Accepted: 29 March 2017/Published online: 27 April 2017

(C) The Author(s) 2018, corrected publication June 2018

\begin{abstract}
Background Distal Doppler-guided transanal hemorrhoidal dearterialization with mucopexy (Doppler-guided THD) seems to be associated with better short-term outcomes than conventional hemorrhoidectomy, but there are little data about long-term recurrence. The aim of this study was to compare Doppler-guided THD for grade III-IV hemorrhoids with conventional hemorrhoidectomy with regard to longterm postoperative morbidity and recurrence.

Methods This was a single-center longitudinal and comparative study of a cohort of patients who underwent either distal Doppler-guided THD with low ligation of the hemorrhoidal artery and mucopexy or conventional excisional hemorrhoidectomy (Milligan and Morgan or Ferguson) for grade III and IV hemorrhoids. Short- and long-term postoperative morbidity was recorded. Severity of hemorrhoid symptoms (bleeding, prolapse, manual reduction, discomfort or pain and impact on quality of life) and fecal continence status (Vaizey score) were evaluated before surgery and at minimum of 1 year after surgery.

Results Eighty-three patients were included in the study. Forty-nine patients (59\%) underwent Doppler-guided
\end{abstract}

The original version of this article was revised due to a retrospective Open Access order.

S. Biondo

sbn.biondo@gmail.com

1 Colorectal Unit, Department of General and Digestive Surgery, Bellvitge University Hospital, University of Barcelona, C/Feixa Llarga s/n, L'Hospitalet de Llobregat, 08907 Barcelona, Spain

2 IDIBELL (Bellvitge Biomedical Investigation Institute), Barcelona, Spain

3 Department of General and Digestive Surgery, Reina Sofia de Cordoba University Hospital, Córdoba, Spain
THD, and $34(41 \%)$ patients underwent conventional hemorrhoidectomy. The 30-day postoperative surgical morbidity was $26.5 \%$ in the Doppler-guided THD group and $8.82 \%$ in the conventional hemorrhoidectomy group $(p=0.085)$. No significant differences between the groups were observed in terms of persistence of bleeding, prolapse, need for manual reduction in prolapse and pain. One (2\%) patient in the THD group and $2(5.4 \%)$ patients in the conventional hemorrhoidectomy group needed further surgical procedures. Minor fecal incontinence occurred only after conventional hemorrhoidectomy in 2 (5.4\%) patients.

Conclusions Our results showed that Doppler-guided THD is not inferior to conventional excisional hemorrhoidectomy for advanced hemorrhoidal disease in terms of postoperative complications and long-term recurrence of symptoms.

Keywords Doppler-guided THD · Hemorrhoidectomy · Dearterialization $\cdot$ Mucopexy $\cdot$ Long-term results

\section{Introduction}

Hemorrhoids are the most common anal pathology and constitute a major socioeconomic and medical problem. It is estimated that $90 \%$ of the general population suffers from hemorrhoidal symptoms at least once in their life [1-3]. Therapeutical options for symptomatic hemorrhoids vary from medical treatment for mild cases to surgical procedures for severe cases or those which fail nonoperative treatment [3]. Although conventional excisional hemorrhoidectomy techniques $[4,5]$ are considered the gold standard for the treatment of grade III and IV hemorrhoids, minimally invasive procedures have been introduced into 
clinical practice in order to reduce postsurgical pain and postsurgical functional limitations [6].

Distal Doppler-guided transanal hemorrhoidal dearterialization associated with mucopexy (Doppler-guided THD) is a recently introduced nonexcisional surgical technique for treating hemorrhoids [7, 8].

Doppler-guided THD seems to be associated with less postsurgical pain, a shorter hospital stay and an earlier return to normal life than conventional hemorrhoidectomy procedures such as Milligan-Morgan or Ferguson hemorrhoidectomy [9-14].

Evidence about long-term outcomes in terms of recurrence and chronic complications after surgery for grade III and IV hemorrhoids is scarce due to the heterogeneity of the studies available. A high long-term symptomatic recurrence rate has been described after conventional hemorrhoidectomy $(20-40 \%)[15,16]$. Some studies report noninferior or better results for Doppler-guided THD in terms of long-term recurrence compared to conventional hemorrhoidectomy techniques, but results are difficult to compare due to their different inclusion criteria and methods for assessing recurrence $[1,11,13,17,18]$.

The aim of this study was to compare the long-term results of Doppler-guided THD for grade III-IV hemorrhoids and conventional hemorrhoidectomy (Milligan and Morgan or Ferguson techniques).

\section{Materials and methods}

This was a single-center, longitudinal and comparative study of a cohort of patients treated for grade III-IV hemorrhoids at the Colorectal Unit of the Bellvitge University Hospital (Barcelona, Spain) between January 2010 and December 2015. Approval for the study was obtained from the hospital ethics committee (PR030/16), and written and verbal informed consent was obtained from all patients. All data were collected and registered prospectively as consecutive cases in an electronic database. Preoperative assessment, diagnosis and classification of hemorrhoids were made by a colorectal surgeon from our center on the basis of physical examination, and anoscopy or proctoscopy in the outpatients' clinic. The Goligher classification was used to establish the grade of hemorrhoids [8]. Colonoscopy was recommended before surgery to rule out colorectal polyps or cancer depending on the patient's age, family history and symptoms.

All patients with grade III and IV hemorrhoids in a minimum of 2 anal quadrants who underwent Dopplerguided THD with mucopexy (Group I) or conventional excisional hemorrhoidectomy (Milligan and Morgan or Ferguson) (Group II) in the study period were included in the analysis. The exclusion criteria were: age younger than
18 years, hemorrhoids treated using other procedures (rubber band ligation, sclerotherapy, stapled hemorrhoidectomy), those with altered cognitive status, inflammatory bowel disease, previous fecal incontinence (Vaizey score $\geq 5$ ) and/or other associated anorectal pathology that could alter the evaluation of the outcomes. The first 15 patients operated on with Doppler-guided THD were also excluded.

All patients were prepared preoperatively with two enemas. Both procedures were performed under general or spinal anesthesia according to anesthesiologist and/or patient preference, with the patient in the lithotomy position. The doppler-guided THD procedure was performed using the THD kit (THD Italy, Corregio, Italy) as described by Ratto et al. $[8,19]$. Using the Doppler device of the THD Slide proctoscope, the 6 hemorrhoidal arteries were identified, the proctoscope was moved distally, and when the best Doppler signal was obtained (usually within the most distal $2 \mathrm{~cm}$ of the rectum), a small 'marker point' was placed on the mucosa using electrocautery. Arteries were ligated separately at 1,3, 5, 7, 9, 11 o'clock of the rectal circumference marking the arterial pulse and including it in a pexy starting all the way inside the rectum about $2 \mathrm{~cm}$ above the anorectal junction with a transfixing mucosal and submucosal stitch. The mucopexy was performed under direct vision with a continuous suture including prolapsing mucosa and submucosa in a proximal-to-distal direction up to $1 \mathrm{~cm}$ from the dentate line. Conventional hemorrhoidectomy was performed as described by Milligan and Morgan [5] or Ferguson [20].

All surgical procedures were performed by experienced surgeons specialized in coloproctology. Patients that underwent Doppler-guided THD were operated on by the same surgeon (SB). In the absence of postoperative complications or difficult pain control, the patients were discharged home the same day of the surgery or after $24 \mathrm{~h}$ depending on associated comorbidities and/or social problems. Patients were recommended to adopt a fiber-rich diet in order to avoid fecal impaction. A standardized analgesic protocol with nonsteroidal anti-inflammatory drugs and acetaminophen was recommended for the first 3 days after surgery. After postoperative day 3, patients were advised to reduce the analgesic doses depending on the severity of the pain. Patients were followed up in the outpatient clinic 30 days after surgery and at 1 year.

Short- and long-term postoperative morbidity was reviewed by medical report and by the prospective database of the colorectal unit. The Clavien-Dindo system was used to classify surgical complications [21].

Baseline and recurrent hemorrhoid symptoms were evaluated prospectively before surgery and at 1 year after surgery by a specific questionnaire designed by Giordano et al. [22]. The questionnaire assesses 5 different 
parameters (bleeding, prolapse, manual reduction, discomfort or pain and impact on quality of life), each scoring from 0 to 4 with 0 corresponding to no symptoms at all and 4 to the presence of the symptoms on a daily basis or with every bowel movement. A total score of 0 corresponded to the complete absence of hemorrhoid symptoms, while a total score of 20 corresponded to the greatest possible intensity of symptoms (Table 1).

The fecal continence status before surgery and at minimum of 1 year after surgery was measured by the Vaizey score [23]. Need for further surgery for persisting or recurring hemorrhoid symptoms and surgery-related complications during follow-up were recorded.

\section{Statistical analysis}

Quantitative data are presented as mean and standard deviation or median and interquartile range. Qualitative data are presented as absolute numbers and percentages. Differences between groups were evaluated using a parametric or nonparametric test as appropriate. Qualitative variables were analyzed using the Chi-square test. Quantitative variables were analyzed using Student's $t$ test or the Mann-Whitney $U$ test. The statistical analysis was performed using Software R 3.3.2 (R Foundation for Statistical Computing, Vienna, Austria), and significance was set at a $p$ value of $<0.05$.

\section{Results}

Between 2010 and 2015, 230 patients were treated for hemorrhoidal disease at Bellvitge University Hospital. Eighty-three patients met the inclusion SEE P.4 criteria and were included in the study analysis (Fig. 1). Baseline characteristics of the patients are shown in Table 2.
Forty-nine patients (59\%) underwent the Doppler-guided THD procedure (Group I), and $34(41 \%)$ patients underwent conventional excisional hemorrhoidectomy (Group II). In Group I, all patients underwent complete circumferential mucopexy. In Group II, 21 patients (61.8\%) underwent Ferguson hemorrhoidectomy and 13 patients (38.2\%) underwent Milligan and Morgan hemorrhoidectomy. All excisional hemorrhoidectomy was performed using electrocautery. Nineteen patients (23.8\%) underwent an additional procedure during the same surgery: in Group I, 13 patients underwent skin tag excision and 4 underwent anal polyp excision, while in Group II, 3 patients underwent an additional rubber banding procedure.

\section{Postoperative morbidity}

No intraoperative complications were observed in either group. The 30-day postoperative surgical morbidity was $26.5 \%$ in Group I and $8.82 \%$ in Group II $(p=0.085)$. Postoperative complications are listed in Table 3.

\section{Long-term outcomes}

Five patients were lost to follow-up and not available for long- term evaluation ( 4 in Group I and 1 in Group II). The mean follow-up period was 1.9 years $(\mathrm{SD} \pm 1.4)$ : 1.13 years $(\mathrm{SD} \pm 0.40)$ for Group $\mathrm{I}$ and 2.89 years $(\mathrm{SD} \pm 1.63)$ for Group II.

During follow-up, in Group I, 1 patient underwent excision for single pile prolapse and 2 patients are on the waiting list for excision of symptomatic persistent external hemorrhoids.

Long-term symptom recurrence was evaluated using the questionnaire shown in Table 1. No significant differences were observed between the mean baseline and last total score between the 2 groups (Table 4; Fig. 2).

Table 1 Hemorrhoid symptom questionnaire score [22]

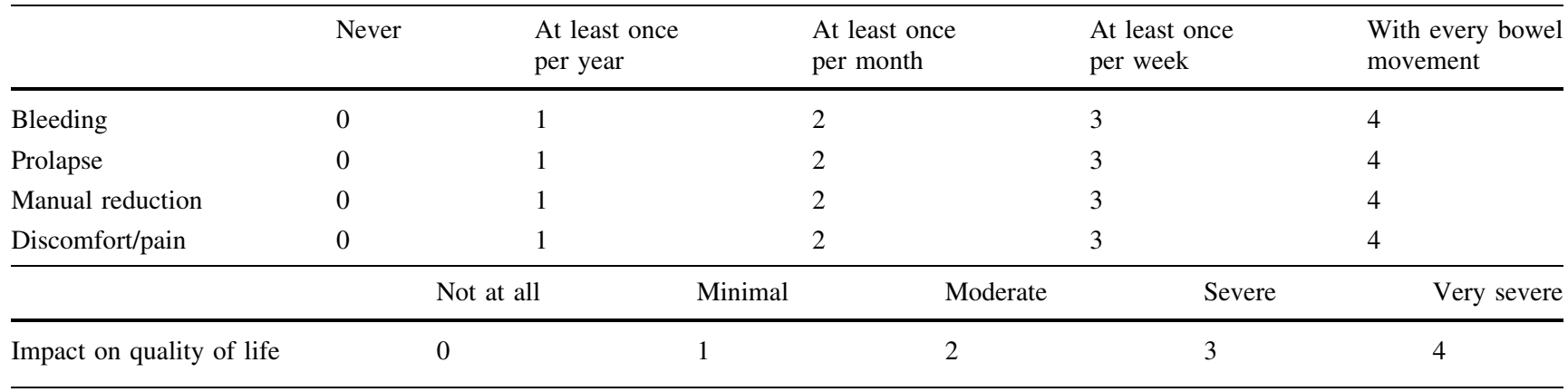


Fig. 1 Flowchart of the study cohort

Patients evaluated for the study $n=230$

- Grade I-II hemorrhoids, single pile grade III-IV

hemorrhoids and ambulatory treatment (banding, sclerotherapy) $n=73$

- Other surgical techniques $n=32$

- Other associated colorectal pathology $n=27$

- Training THD procedure cases $n=15$

\section{3 patients fit the inclusion criteria}

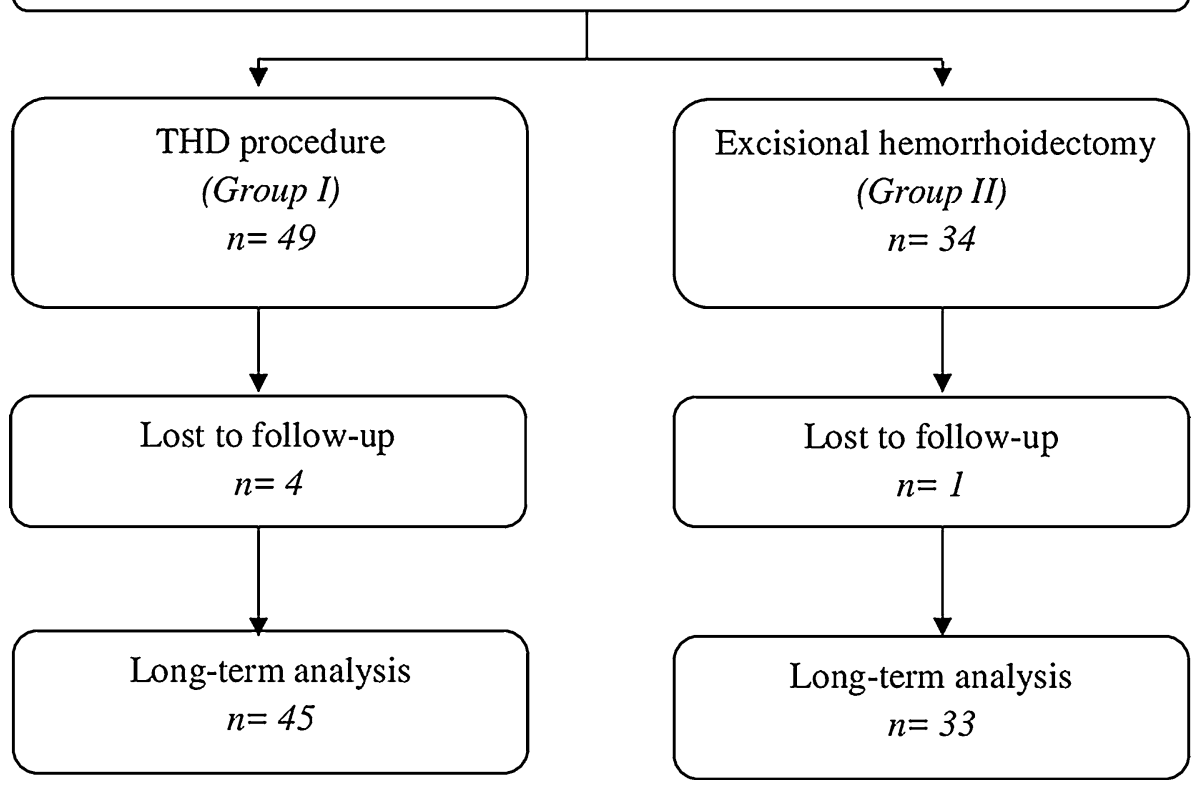

Table 2 Baseline characteristics of patients

\begin{tabular}{lccr}
\hline & $\begin{array}{l}\text { All patients } \\
n=83\end{array}$ & $\begin{array}{l}\text { THD procedure } \\
\text { (Group I) } n=49\end{array}$ & $\begin{array}{l}\text { Excisional hemorrhoidectomy } \\
\text { (Group II) } n=34\end{array}$ \\
\hline $\begin{array}{l}\text { Age, years (mean, SD) } \\
\text { Gender }\end{array}$ & $55.4(11.4)$ & $53.8(11.6)$ & $57.7(10.9)$ \\
Male & $52(62.7 \%)$ & $28(57.1 \%)$ & $24(70.6 \%)$ \\
Female & $31(37.3 \%)$ & $21(42.9 \%)$ & $10(29.4 \%)$ \\
Goligher classification & & & 0.310 \\
III & $47(56.6 \%)$ & $27(55.1 \%)$ & $20(58.8 \%)$ \\
IV & $36(43.4 \%)$ & $22(44.9 \%)$ & $14(41.2 \%)$ \\
Recurrent hemorrhoids & $4(4.8 \%)$ & $1(3.12 \%)$ & $3(6.12 \%)$ \\
Hemorrhoid symptomatology score (mean, SD) & $11.8(3.26)$ & $11.7(2.93)$ & $11.9(3.73)$
\end{tabular}

Analyzing separately grade III and IV hemorrhoids, there was no difference in the results for patients with grade IV hemorrhoids, while for patients with grade III hemorrhoids, the THD procedure appears to better reduce the total symptoms score than excisional hemorrhoidectomy (Table 4; Fig. 2).

No statistical differences between the groups were observed in terms of persistence of some degree of 
Table 3 Postoperative morbidity

\begin{tabular}{lll}
\hline Type of complication (related treatment) & Group I $(n)$ & Group II $(n)$ \\
\hline Acute urinary retention (bladder catheterization) & 7 & 1 \\
Hemorrhoid thrombosis (thrombectomy under local anesthesia) & 4 & - \\
Acute bleeding (hemostasis UA) & - & 1 \\
Perianal/anal infection (debridement UA plus antibiotic) & 1 & - \\
Perianal/anal infection (antibiotics) & 1 & 1 \\
\hline
\end{tabular}

$n$ number of complications

Group I THD procedure; Group II conventional excisional hemorrhoidectomy; UA under anesthesia

Table 4 Long-term follow-up of the hemorrhoid symptoms

\begin{tabular}{|c|c|c|c|c|c|c|c|c|c|}
\hline \multirow{2}{*}{$\begin{array}{l}\text { Hemorrhoid } \\
\text { symptoms score }\end{array}$} & \multicolumn{3}{|c|}{ Grade III-IV hemorrhoids } & \multicolumn{3}{|c|}{ Grade III hemorrhoids } & \multicolumn{3}{|c|}{ Grade IV hemorrhoids } \\
\hline & $\begin{array}{l}\text { Group I } \\
n=45\end{array}$ & $\begin{array}{l}\text { Group II } \\
n=33\end{array}$ & $p$ & $\begin{array}{l}\text { Group I } \\
n=24\end{array}$ & $\begin{array}{l}\text { Group II } \\
n=19\end{array}$ & $p$ & $\begin{array}{l}\text { Group I } \\
n=21\end{array}$ & $\begin{array}{l}\text { Group II } \\
n=14\end{array}$ & $p$ \\
\hline \multicolumn{10}{|c|}{ Total score mean (SD) } \\
\hline Basal & $11.6(2.93)$ & $12.0(3.75)$ & 0.574 & $11.6(2.92)$ & $12.1(4.14)$ & 0.679 & $11.5(3.01)$ & $11.9(3.29)$ & 0.715 \\
\hline Last follow-up & $1.24(2.39)$ & $1.52(2.28)$ & 0.613 & $0.79(1.59)$ & $1.74(2.77)$ & 0.196 & $1.76(3.02)$ & $1.21(1.42)$ & 0.477 \\
\hline \multicolumn{10}{|c|}{ Differential $^{a}$ mean (SD) } \\
\hline Value & $-10.31(3.29)$ & $-10.48(4.70)$ & 0.856 & $-10.79(3.19)$ & $-10.32(5.41)$ & 0.737 & $-9.76(3.40)$ & $-10.71(3.71)$ & 0.449 \\
\hline Percentage & $-90.01(18.2)$ & $-84.52(25.2)$ & 0.292 & $-93.23(12.9)$ & $-81.42(31.19$ & 0.135 & $-86.32(22.6)$ & $-88.71(13.8)$ & 0.700 \\
\hline
\end{tabular}

Group I THD procedure; Group II conventional excisional hemorrhoidectomy

${ }^{a}$ Differential has been calculated subtracting the value of the total last follow-up score from the total baseline score; only patients with completed follow-up data are considered for the analysis

bleeding, prolapse, need for manual reduction in prolapse, pain and quality of life impairment (Table 5).

No patients in Group I reported fecal incontinence, though 2 women, 58 and 47 years old, reported persistent postsurgical urgency of defecation at the last follow-up. In Group II, 2 patients reported fecal incontinence as a consequence of surgery. The first one was a 62-year-old male with a preoperative Vaizey score of 4 , due to occasional incontinence to liquid stool, but 3 years after surgery a Vaizey score of 14, with monthly episodes of incontinence for solid and liquid stool, difficulty in flatus control and the need to wear pads. No other events happened which could justify this deterioration in his fecal continence. This patient refused any other treatment because the impact of the symptoms on his quality of life was low. The second patient was a 43-year-old woman without a history of previous fecal incontinence, but 1 year after surgery persistent occasional incontinence to liquid and solid stool with a Vaizey score of 6. Biofeedback therapy was indicated.

In Group I, 1 patient was diagnosed after two months with a submucosal anal fistula that was laid open and another patient was diagnosed with a postoperative anal fissure which responded to topical treatment.

\section{Discussion}

The most important finding of this study is that the Doppler-guided THD procedure is not inferior to conventional excisional hemorrhoidectomy for advanced hemorrhoidal disease in terms of postoperative complications and longterm symptom recurrence.

The major strength of this study is that only patients with grade III and IV hemorrhoids were included. Shortand long-term follow-up was performed by the same surgical team during the study period, and recurrence or persistence of hemorrhoid symptoms was evaluated using a symptomatic score that takes into account bleeding, prolapse, pain and impact of hemorrhoidal disease on quality of life. Clinical evaluation using only the Goligher grade could cause confusion regarding true symptomatic recurrence or symptom persistence, the elimination of which must be considered the real goal of surgery for hemorrhoids.

The study has some limitations. First, the number of patients included was relatively small due to the restricted inclusion criteria, which may be responsible for a power error. Nevertheless, these were consecutively enrolled patients, and there were no differences in demographics 


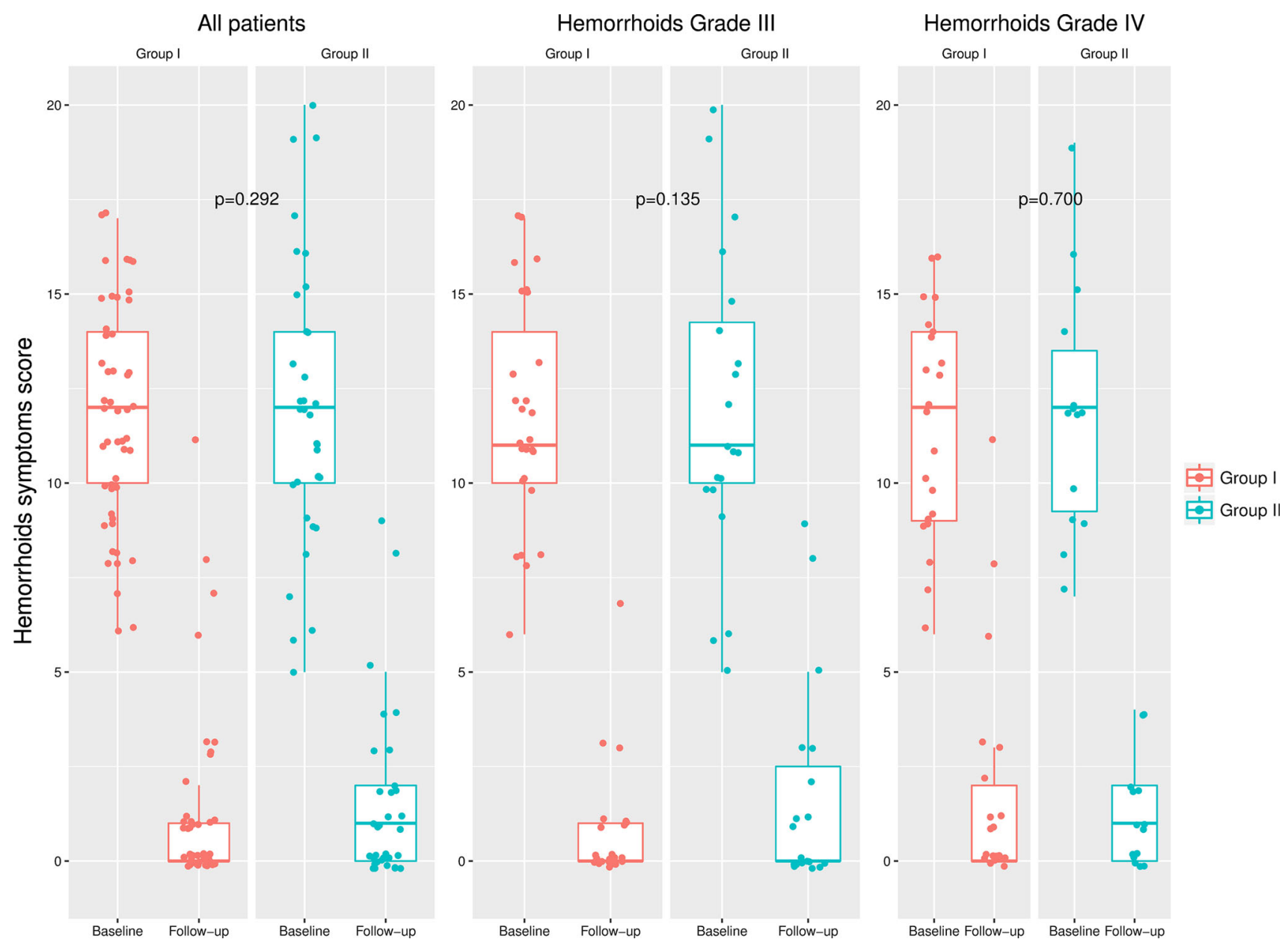

Fig. 2 Boxplot of basal and long-term symptoms score according to the surgical techniques and hemorrhoids grade. Group I Doppler-guided THD procedure; Group II conventional excisional hemorrhoidectomy

Table 5 Long-term symptom persistence

Patients with persistent symptoms at last follow-up/patients with symptoms at baseline (\%)

\begin{tabular}{lllr}
\hline & THD procedure (Group I) & Excisional hemorrhoidectomy (Group II) & $p$ \\
\hline Bleeding & $9 / 42(21.4)$ & $9 / 28(32.1)$ & 0.468 \\
Prolapse & $7 / 44(15.9)$ & $3 / 32(9.4)$ & 0.505 \\
Manual reduction & $1 / 25(4)$ & $1 / 19(5.3)$ & 1.000 \\
Discomfort/pain & $6 / 35(17.1)$ & $7 / 31(22.6)$ & 0.807 \\
Impairment of quality of life & $3 / 39(7.7)$ & $6 / 31(19.4)$ & 0.171 \\
\hline
\end{tabular}

Percentages are calculated considering patients that presented symptoms of any degree or frequency at the baseline and at the last follow-up

and disease grade between the 2 groups. Second, a classic well-known surgical technique (hemorrhoid excision) was compared with Doppler-guided THD which is a relatively new procedure. This could introduce a bias in the analysis of the results. Actually, as recently observed [24], the number of complications can decrease with increasing surgeon experience. To reduce this error, before starting the inclusion of patients for the study, the Doppler-guided THD procedure was performed on 15 patients. Moreover, it was performed only by 1 surgeon. Another weakness of the present study is that postoperative pain has not been studied. Considering that most of the published studies aim to analyze the relationship between type of procedure and postoperative pain, we focussed our analysis on early 
complications and long-term results. In addition, postoperative pain is a difficult point to study outside of a targeted randomized trial, because it depends on many variables which are not directly related to the surgical technique such as pain medication protocol, anesthesia and scales used to record the pain. In a recent double-blind randomized controlled trial comparing 20 patients who underwent Doppler-guided THD with mucopexy with 20 patients who underwent conventional hemorrhoidectomy, the authors found that the dearterialization led to less postoperative pain [12]. Other authors found that pain was similar after both surgical techniques $[11,13]$.

As reported in others studies [11-13, 25], we did not find statistical differences in overall postoperative morbidity between the two techniques. Nevertheless, a higher number of acute urinary retention occurred after Dopplerguided THD. This is not a major complication and all the cases were effectively treated by a temporary bladder catheterization removed the same day or the day after surgery. However, frequently this mild complication implied a delay in hospital discharge. Four patients experienced acute internal hemorrhoid thrombosis during the first 30 days after Doppler-guided THD. All cases were treated successfully by thrombectomy under local anesthesia with immediate relief of pain. While uncommon after excisional hemorrhoidectomy, thrombosis needs to be suspected when a patient presents acute anal pain in the first weeks after a THD procedure.

A recent meta-analysis including 316 patients from 4 randomized controlled trials, which evaluate the surgical and postoperative outcomes of Doppler-guided THD procedure versus open conventional hemorrhoidectomy, finds no differences in terms of recurring disease between the surgical procedures [25]. These results are in line with our findings in which long-term symptom recurrence or persistence was similar in the 2 groups. However, when a separate analysis was performed for different grades of hemorrhoids, for grade III the Doppler-guided THD procedure appears to be more effective in long-term symptom control compared to excisional hemorrhoidectomy, although this result did not reach statistical significance. This could be explained by a minor effect of the mucopexy on grade IV hemorrhoids in which the chronic prolapsed piles are often epithelialized making fibrosis less effective in maintaining the piles in the anal canal. The same trend was observed by Denoya et al. in a recent randomized double-blinded trial comparing Doppler-guided THD with excisional hemorrhoidectomy, in which all patients who experienced recurrence in either arms had grade IV hemorrhoids [17].

Analyzing separately the symptoms included in the Giordano score, no differences between the 2 groups were observed in terms of persistence of symptoms. Although the rate of persistence of symptoms is apparently high, in most patients, symptoms were occasional and therefore had a low clinical impact. Actually, only 3 patients included in the study have been considered for further surgical treatment.

Long-term chronic complications such as anal stricture, nonhealing wounds and fecal incontinence as consequence of sphincter lesions have been reported after conventional excisional hemorrhoidectomy but not after Doppler-guided THD [16, 17]. In the present study, we did not observe any anal stricture or chronic nonhealing wounds, but 2 patients reported fecal incontinence after excisional hemorrhoidectomy. One of these patients had previous mild continence impairment that worsened after surgery. Due to the fact that sphincter lesions are only anecdotal after Doppler-guided THD, this surgical technique should be preferred for patients at high risk of fecal incontinence.

Operation time was not analyzed in the present study. As reported in several publications, the operation time for the Doppler-guided THD procedure is longer than for excisional hemorrhoidectomy $[13,25]$. This disadvantage must be balanced with the fact that Doppler-guided THD is less invasive than conventional hemorrhoidectomy because no wounds are created and there is no risk of sphincter damage. Another potential advantage of Doppler-guided THD, though time-demanding, is that it can correct the physiology of the hemorrhoidal plexus by of the arterial blood flow to the hemorrhoidal cushions through dearterialization and eliminate the mucosal prolapse by mucopexy in 6 different points of the anal canal [22, 26, 27].

\section{Conclusions}

Our results showed that Doppler-guided THD is a safe and effective technique for treating grade III-IV symptomatic hemorrhoids. When compared to conventional excisional hemorrhoidectomy, results in terms of symptom recurrence or persistence at long-term follow-up are similar. These results need to be validated in large and multicenter randomized trials to elucidate whether Doppler-guided THD procedure is truly better than conventional hemorrhoidectomy.

Acknowledgements The authors thank Mr Bernat Miguel, Data Manager of the Colorectal Unit, University Hospital of Bellvitge and IDIBELL, for the statistical analysis.

\section{Compliance with ethical standards}

Conflict of interest Sebastiano Biondo is a trainer for the Dopplerguided THD procedure. The other authors have no conflicts of interest. 
Ethical approval Approval for the study was obtained from the hospital ethics committee (PR030/16).

Informed consent Written and verbal informed consent was obtained from all patients.

Open Access This article is distributed under the terms of the Creative Commons Attribution 4.0 International License (http://crea tivecommons.org/licenses/by/4.0/), which permits use, duplication, adaptation, distribution and reproduction in any medium or format, as long as you give appropriate credit to the original author(s) and the source, provide a link to the Creative Commons license and indicate if changes were made.

\section{References}

1. Zampieri N, Castellani R, Andreoli R, Geccherle A (2012) Longterm results and quality of life in patients treated with hemorrhoidectomy using two different techniques: ligasure versus transanal hemorrhoidal dearterialization. Am J Surg 204:684-688

2. Johanson JF, Sonnenberg A (1990) The prevalence of hemorrhoids and chronic constipation. an epidemiologic study. Gastroenterology 98:380-386

3. Madoff RD, Fleshman JW, Clinical Practice Committee, American Gastroenterological Association (2004) American Gastroenterological Association technical review on the diagnosis and treatment of hemorrhoids. Gastroenterology 126:1463-1473

4. Ferguson JA, Mazier WP, Ganchrow MI, Friend WG (1971) The closed technique of hemorrhoidectomy. Surgery 70:480-484

5. Milligan E, Morgan C, Jones L, Officer R (1937) Surgical anatomy of the anal canal and the operative treatment of hemorrhoids. Lancet 2:1119-1124

6. Joshi GP, Neugebauer EAM, PROSPECT Collaboration (2010) Evidence-based management of pain after haemorrhoidectomy surgery. Br J Surg 97:1155-1168

7. Dal Monte PP, Tagariello C, Sarago M et al (2007) Transanal haemorrhoidal dearterialisation: nonexcisional surgery for the treatment of haemorrhoidal disease. Tech Coloproctol 11:333-338 (discussion 338-9)

8. Ratto C (2014) THD Doppler procedure for hemorrhoids: the surgical technique. Tech Coloproctol 18:291-298

9. Infantino A, Bellomo R, Dal Monte PP et al (2010) Transanal haemorrhoidal artery echodoppler ligation and anopexy (THD) is effective for II and III degree haemorrhoids: a prospective multicentric study. Colorectal Dis 12:804-809

10. Giordano P, Tomasi I, Pascariello A, Mills E, Elahi S (2014) Transanal dearterialization with targeted mucopexy is effective for advanced haemorrhoids. Colorectal Dis 16:373-376

11. De Nardi P, Capretti G, Corsaro A, Staudacher C (2014) A prospective, randomized trial comparing the short- and long-term results of doppler-guided transanal hemorrhoid dearterialization with mucopexy versus excision hemorrhoidectomy for grade III hemorrhoids. Dis Colon Rectum 57:348-353

12. Denoya PI, Fakhoury M, Chang K, Fakhoury J, Bergamaschi R (2013) Dearterialization with mucopexy versus haemorrhoidectomy for grade III or IV haemorrhoids: short-term results of a double-blind randomized controlled trial. Colorectal Dis 15:1281-1288

13. Elmér SE, Nygren JO, Lenander CE (2013) A randomized trial of transanal hemorrhoidal dearterialization with anopexy compared with open hemorrhoidectomy in the treatment of hemorrhoids. Dis Colon Rectum 56:484-490

14. Ratto C, Giordano P, Dionisi L, Parello A, Litta F, Doglietto G (2011) Transanal haemorrhoidal dearterialization (THD) for selected fourth-degree haemorrhoids. Tech Coloproctol 15:191-197

15. Konsten J, Baeten CG (2000) Hemorrhoidectomy vs. Lord's method: 17-year follow-up of a prospective, randomized trial. Dis Colon Rectum 43:503-506

16. Qarabaki MA, Mukhashavria GAGGA, Mukhashavria GAGGA, Giorgadze NG (2014) Circular vs. three-quadrant hemorrhoidectomy for end-stage hemorrhoids: short- and long-term outcomes of a prospective randomized trial. J Gastrointest Surg 18:808-815

17. Denoya P, Tam J, Bergamaschi R (2014) Hemorrhoidal dearterialization with mucopexy versus hemorrhoidectomy: 3-year follow-up assessment of a randomized controlled trial. Tech Coloproctol 18:1081-1085

18. Bursics A, Morvay K, Kupcsulik P, Flautner L (2004) Comparison of early and 1-year follow-up results of conventional hemorrhoidectomy and hemorrhoid artery ligation: a randomized study. Int J Colorect Dis 19:176-180

19. Ratto C, Dionisi L, Parello A, Litta F, Zaccone G, De Simone V (2012) "Distal Doppler-guided dearterialization" is highly effective in treating haemorrhoids by transanal haemorrhoidal dearterialization. Colorect Dis 14:e786-e789

20. Ferguson JA, Heaton JR (1959) Closed hemorrhoidectomy. Dis Colon Rectum 2:176-179

21. Dindo D, Demartines N, Clavien P-A (2004) Classification of surgical complications: a new proposal with evaluation in a cohort of 6336 patients and results of a survey. Ann Surg 240:205-213

22. Giordano P, Nastro P, Davies A, Gravante G (2011) Prospective evaluation of stapled haemorrhoidopexy versus transanal haemorrhoidal dearterialisation for stage II and III haemorrhoids: three-year outcomes. Tech Coloproctol 15:67-73

23. Vaizey C, Carapeti E, Chaill J, Kamm M (1999) Prospective comparison of faecal incontinence grading systems. Gut 44:77-80

24. Bjelanovic Z, Draskovic M, Veljovic M, Lekovic I, Karanikolas M, Stamenkovic D (2016) Transanal hemorrhoid dearterialization is a safe and effective outpatient procedure for the treatment of hemorrhoidal disease. Cir Esp 94:588-594

25. Xu L, Chen H, Lin G, Ge Q, Qi H, He X (2016) Transanal hemorrhoidal dearterialization with mucopexy versus open hemorrhoidectomy in the treatment of hemorrhoids: a metaanalysis of randomized control trials. Tech Coloproctol 20:825-833. doi:10.1007/s10151-016-1551-2

26. Morinaga K, Hasuda K, Ikeda T (1995) A novel therapy for internal hemorrhoids: ligation of the hemorrhoidal artery with a newly devised instrument (Moricorn) in conjunction with a Doppler flowmeter. Am J Gastroenterol 90:610-613

27. Ratto C, de Parades V (2015) Doppler-guided ligation of hemorrhoidal arteries with mucopexy: a technique for the future. J Visc Surg 152:s15-s21 